\title{
Spinal cord injuries: the last decade and the next
}

\author{
D J Brown FRACP FACRM
}

Spinal Injuries Unit, Austin Hospital, Heidelberg, Victoria 3084, Australia.

The last decade has seen many changes and challenges. It began with the International Year of the Disabled Person (IYDP) in 1981, which was a landmark year. It signalled the achievement of the major early goals of spinal cord injuries management which were to decrease morbidity and mortality and increase the lifespan of the spinal cord injured to near normal levels.

IYDP can be considered to have started a new era. It prepared the community for the re-emergence of disabled people and it gave expression to the desire of the disabled to become active members of their communities. It also gave greater emphasis to community rehabilitation and allowed professional workers to shift their emphasis from 'rehabilitation in hospital' to 'rehabilitation in the home, community and at work'. Now more than ever before, the institutional programme is seen to be one of skills acquisition in preparation for return home. The goals of rehabilitation have shifted from 'discharge from hospital' to 'the development of a satisfying and productive lifestyle after discharge'.

It is vital therefore, that the rehabilitation facility does not mirror the acute setting with its emphasis on ill health. The message of rehabilitation must be that patients are healthy but disabled. The facility must be more home-like, with greater emphasis on privacy and dignity. This is particularly important to the spinal cord injured, for whom the institutional phase of skills acquisition is prolonged.

The hospital programme continues to be one of physical skills training and theoretical learning, but now places greater emphasis on the redevelopment of life skills in general. The development of psychosocial rehabilitation has placed greater emphasis on helping the patient and family to cope with the disaster of spinal cord injury and to support them in their search for a new and meaningful life. They should no longer be expected to bring light and order to the chaos created by spinal cord injury without the aid of empathic professionals.

The emphasis upon reconstituting the psychological and emotional component of the family group has given greater depth and breadth to professional roles in rehabilitation. ${ }^{1,2}$ It is realised that the staff must be aware of this aspect of the patient's rehabilitation. The need for greater involvement of the psychosocial disciplines in the rehabilitation team has been recognised and means are being found to enable this to occur. The psychosocial disciplines include psychiatry, clinical psychology (including neuropsychology), social work and chaplaincy. These team members usually do not work directly with the patient and family, but very often work through those team members involved in the physical aspects of rehabilitationnurses, physiotherapists and occupational therapists in particular. These hands-on professionals must be alert to the nuances of the patient's mood, must adopt appropriate responses to his attitudes in order to reinforce positive and appropriate behaviour to help the patient to control inappropriate, antisocial and destructive behavioural patterns and must seek psychological help when needed. ${ }^{3}$

This new emphasis has changed hospital rehabilitation in quite a fundamental way. Not only is the rehabilitation team aiming to return a patient to an active community life, as similar as possible to that enjoyed before the disability, but the team is now involved in helping many of the 'socially disadvantaged' patients to develop more appropriate behavioural patterns, social skills and self confidence. This is not rehabilitation in the classical sense. It involves the further development of the individual's emotional maturity and personality in order to produce a more capable and successful individual than 
he was before the accident.

It is apparent that this approach has led to more complete rehabilitation of the patient and family, and has enabled better reintegration into the community. This success is seen in many ways. It includes the greater stability of the patient's marriage and a decreased incidence of readmissions for preventable complications. We have seen a significant fall in the number of grades 3 and 4 pressure sores in our unit, and also a decrease in the size of the sores themselves. This is, in large measure, a result of a much greater psychosocial component of the rehabilitation programme, and also of better community follow up.

The further development of follow up and after care that we have seen in the last decade is a direct result of IYDP placing greater community values on the disabled person. Regular review has been a practice in many spinal units for a long time and some have had visiting nurses. ${ }^{4}$ In the last 10 years many more spinal cord units have nurses visiting patients in the community and liaising with them and their local medical support and also with other community support organisations. Spinal unit follow up has also been linking patients with normal community organisations as well as with those especially set up for disabled people. This has been particularly noticeable in sport, where many disabled people now compete alongside able bodied people, and we have seen it develop in a more concerted way in other areas of social activity. By involving the patients in their communities soon after discharge, they have community support in their struggle to establish a new lifestyle and they more quickly gain positive feedback which emphasises that they are still valuable people.

Many aspects of what might be called later rehabilitation have blossomed. Sexuality of the disabled person has become more accepted as part of rehabilitation both in hospital and particularly after discharge, when patients are very often more ready to tackle this extremely delicate area. In addition more spinal cord injured people are aspiring to become parents and the availability of many techniques to help them achieve this objective has resulted in a steady increase in the number of male patients becoming fathers. ${ }^{5}$ Circumventing many of the barriers to fatherhood has been quite successful, but many male patients are still unable to father children, even with all of the modern techniques available.

Sexual intercourse has also seen many changes. Many methods are available for male patients to overcome their impotence. Papaverine injections into the corpora, vacuum condoms, rings and penile implants have all been used. ${ }^{6,7}$

The management of impotence has been included in sexuality programmes and is seen as part of the broader subject of sexual relationships, rather than a problem in itself. This also indicates a broading and deepening of the rehabilitation of the spinal cord injured patient.

As one might expect, later rehabilitation after return to the community has involved practical issues of a strictly physical nature. Tendon transfers for the quadriplegic patient have become very widespread in recent times. ${ }^{8-10}$ Techniques have improved and the periods of postoperative disability and of hospitalisation have decreased. The success of these transfer operations is seen in increased independence for the quadriplegic patient.

Driving for the high quadriplegic patient has become accepted as more suitable equipment has been developed. This equipment is gradually becoming more available, and the skills to enable the quadriplegic patient to tackle driving are becoming disseminated. There is no doubt that in the next 10 years it will become a regular part of the rehabilitation programme offered to these patients.

Another major change in the field of spinal cord rehabilitation has been the social definition of independence as distinct from the physical one. This defines independence as the patient doing what he wants to, even though it may require the physical help of others to achieve his desire. The problem with attendant care has been funding. This cost has now been accepted by insurance companies and in some instances by Government. This new service group has greatly increased the mobility and community involvement of the more severely dis- 
abled quadriplegic patients and has had an effect on the local community by providing employment. It must be one of our aims in the coming years to foster attendant care so that the severely disabled person can stay out of institutional care, not be a burden on his family and do what he wants to, when he wants to.

We have seen great changes in the management of those scourges of the spinal cord injured patient, urinary problems and pressure sores. Urinary tract infections remain a major cause of morbidity, but the long term complications of bladder and kidney damage seem less.

Intermittent clean self catheterization has become accepted as a method of bladder drainage that is as effective as reflex emptying or abdominal straining. ${ }^{11}$

Percutaneous surgical techniques for removing stones from kidneys have greatly decreased the morbidity of renal operations and have also improved kidney function. ${ }^{12,13}$ Bladder operations such as clam cystoplasty have improved bladder capacity and made many people continent. ${ }^{14}$

Bladder management has been one area where electrical stimulation has proved to be a great advance. The anterior sacral root stimulator developed by Giles Brindley has made many male patients condom free, and enabled many male and female patients to become continent. ${ }^{15,16}$ It has also increased bladder emptying and decreased the incidence of urinary tract infection.

The other great scourge of patients has been pressure sores. Greater emphasis on posture and pressure management has lead to new wheelchair design and in particular, development of a number of improved cushions. These have improved ischial pressure relief and have also contributed to the management of adolescents prone to kyphoscoliosis.

Naturally posture and pressure clinics, wheelchairs and cushions do not prevent pressure sores. Good personal care remains the foundation of skin management. Unfortunately this continues to be a problem with a number of patients and pressure sores are the major cause of morbidity and hospitalisation today. Many new wound dressings have been developed, but none is clearly superior to others in a clinical sense. However, many have great convenience in terms of cost and nursing time taken to dress a wound. Hospitalisation time has been decreased by the greater resort to surgery and in particular to the use of large rotation flaps. For those few patients who are unable to have rotation flaps and yet have the problem of recurrent pressure sores where all subcutaneous tissue has been lost, the carbon fibre implant has proved to be an extremely useful method of providing spread of pressure and a lower incidence of pressure sores. ${ }^{17}$ Nine of our patients have had carbon fibre pads implanted. One became infected and had to be removed in the postoperative period. One patient died later of unrelated causes, but had not had any recurrence of pressure sore in the intervening months. One developed a pressure sore over the implant, which became infected and required to be removed. The remaining 6 have not had a recurrence of a pressure sore in the area of the carbon fibre pad. All 9 patients had had multiple hospital admissions for recurrent pressure sores and had had many operations, leaving then with no possibility for further rotation flaps. A carbon fibre pad was their last resort. It has proved to be extremely successful for most of them.

The anterior sacral root stimulator (vide supra) is the only form of neuromuscular electrical stimulation (NMES) which is functional and therefore useful in clinical practice. Unfortunately electrical stimulation has not proved functional in other areas. Much money and time has been expended on electrical stimulation for walking, but this is still not a practical clinical proposition. However upper limb electrical stimulation for palmar and pinch grasp is now on the verge of clinical usefulness and is likely to become, in the next decade, a major treatment leading to further independence of patients. ${ }^{18}$ Its greatest usefulness will be in the C5 quadriplegic patient who has little prospect for tendon transfer other than deltoid-to-triceps. In $\mathrm{C} 6$ and some $\mathrm{C7}$ quadriplegics it will complement tendon transfers.

We have seen many changes in the treatment of spasm. Diazepam, baclofen 
and dantrolene have been complemented by clonidine $^{19}$ and cyproheptadine. ${ }^{20}$ Motor point and nerve blocks have been extended to new areas.

The intrathecal pump has found favour in many units. ${ }^{21}$ Morphine or baclofen are used in small doses to dampen spasm at its source in the spinal cord. Problems with the supply of baclofen have been of concern in Australia and possibly also in other countries. Though it will only occupy a small niche in spasm management, the intrathecal pump is a useful advance.

The new diagnostic modality of magnetic resonance imaging has promised to revolutionise our early management. ${ }^{22-24}$ It will help to define more clearly the indications and types of operation which may benefit the injured spinal cord. It is the preferred investigation for the diagnosis of post traumatic syrinx. $^{25}$

Orthopaedic management has undergone considerable change. The importance of instrumenting as few spinal segments as necessary and of maintaining the normal contour of the spine, especially lumbar lordosis, has been recognised. Transpedicular fixation, in particular of the thoracolumbar spine, is now the preferred method of posterior stabilisation in most cases. ${ }^{26}$ The internal skeletal fixation systems, in addition to providing rigid fixation over a few segments, have the ability to distract axial compression burst fractures or to compress distractive injuries of the thoracolumbar spine.

Posterior stabilisation of the cervical spine can now be achieved by facet screws, laminar clamps or transpedicular screw plate systems.

Type II odontoid fractures are now stabilised, either acutely or in cases of non union, by 2 screws entering the body of the axis anteriorly at the $\mathrm{C} 2 / 3$ disc junction.

Anterior stabilisation is less often used than the posterior approach in spinal trauma. Corpectomy and interbody graft may be supplemented by a screw plate system or, in the thoracolumbar spine, by a screw rod system.

Patients with an incomplete neurological lesion and MRI evidence of continued neural compression, benefit from decom- pression by anterior corpectomy and interbody fusion. ${ }^{27}$ Adequate restitution of canal size can be achieved by a posterior laminectomy within 2 weeks of a cauda equina lesion. ${ }^{28}$

Halo thoracic stabilization devices have been much improved and MRI compatible ones are now available. New plastics have made it possible to use cast jackets for thoracolumbar bracing and cast braces have vastly improved the management of lower femoral and tibial fractures, allowing the patient to return home quickly with stability of the fracture and protection of the skin.

Research has continued to be a major focus in a number of laboratories, and is often sponsored by patient organisations. Restoration of function has not yet occurred, and research into cord regeneration has not provided any treatment of clinical usefulness at this stage. However progress has been made in the prevention of secondary metabolic damage after the acute spinal cord injury. In 1990 a multicentre trial undertaken in the United States of America showed that methylprednisolone when given within 8 hours of injury leads to improved neurological outcome. ${ }^{29}$ It is hoped that clinical application of this drug as soon as possible after injury will lead to an alteration of the patient's neurological outcome so that we will see a change in the disabilities of our patient population.

Recently another paper has suggested that GM-1 ganglioside may be helpful in the early management of the acute spinal cord injury, again with improvement in the neurological outcome. ${ }^{30}$ Whether these neurological improvements will be associated with significant functional improvement remains to be seen.

It has surely been one of the challenges of the last 10 years to devise better methods of functional assessment. The improvement of the Barthal index by modification into the functional independence measure (FIM) has gone closer to this goal than has any previous attempt. We need a reliable useful measure of function in order to be able to assess improvement and in order to be able to communicate with each other regarding the outcomes of our rehabilitation programmes. ${ }^{31}$ 
Now that our programmes focus on improved lifestyles, we are obviously in need of a quality of life measure that we can use to assess the success of our rehabilitation programmes in the community. It will be a major challenge in the next 10 years to devise adequate quality of life assessment measures.

Focus on psychosocial rehabilitation will be helpful here. The quality of life measures used in other areas of medicine that are not traditionally considered rehabilitation, such as post transplant patients, may be helpful in guiding us to a clinically useful measure.

Although restoration of voluntary walking by electrical stimulation or cord regeneration have not proved possible, we have seen some improvement in detail in the development of new walking devices such as the HGO (paraWalker) and the RGO. ${ }^{32}$ These have found greater acceptance than the old caliper and crutch systems, largely because of the lower energy costs for walking. Unfortunately neither is perfect and most patients still prefer their wheelchairs.

The next decade will provides us with significant new challenges. I have mentioned some already. Others will come from changing economic and political situations. The need for spinal cord units is being increasingly recognised and has led to the establishment of new units. ${ }^{33,34}$ Those working from established spinal cord units will be increasingly asked to help with the establishment of units in other parts of the world, so that no spinal cord paralysed patient will go without proper care. The International Medical Society of Paraplegia is one organisation which has a declared aim of improving spinal cord injury care throughout the world and has actively supported increased availability of care and improved quality of care in nations which have few or no spinal cord units. In those countries with an adequate number of spinal cord units, emphasis will be on increasing the availability of quality care and treatment for patients with medical causes of spinal cord damage so that they do not continue to suffer as poor cousins of those who have acquired their disability through trauma. The resources of the spinal cord unit are less available to them, often because they are not referred, and they do not have such extensive insurance cover as their traumatic counterparts.

As the cost of medicine is scrutinised more carefully, we will have a leading role in the prevention of traumatic spinal cord injury. This will involve the evaluation of driver attitudes and training programmes, and also promotion of better road and car designs. These measures must, from our point of view, be seen to be as important in medicine as environmental conservation is to the community at large. It will be on our shoulders to promote preventive medicine as the most cost effective approach for the community. This approach will often cross government departmental boundaries and will require us to persuade the bureaucrats that they need to take a broader view of health care. For example, in order to decrease the amount of money that governments spend on invalid pensions, it will be necessary to prevent people from becoming invalid and therefore the Social Security Department that is likely to save money in the future may need to be involved in the prevention of illness in conjunction with the Health Department whose traditional role this is.

The economic strictures that we now face are likely to continue and our challenge is to maintain the standard of care in our units to the highest possible level. This means fighting for an appropriate share of the medical money available in order that our patients may have the best opportunity to develop satisfying lifestyles and to help them to remain free of preventable complications. This is important if they are to play an active role in society and contribute to the community they live in. Anything less than this is a misuse of limited resources and does neither us nor the community any credit. We must continue to strive for excellence.

\section{References}

1 Judd FK, Brown DJ (1987) Psychiatry in the spinal injuries unit. Paraplegia 25: 254-257. 
2 Judd FK, Brown DJ (1988) The psychosocial approach to rehabilitation of the spinal cord injured patient. Paraplegia 26: 419-424.

3 Judd FK, Stone BA, Webber JE, Brown DJ, Burrows GD (1989) Depression following spinal cord injury. Br J Psychiatry 154: 668-671.

4 Beer NIE (1984) The role of the home visiting nurse in the total education programme of spinal cord injured persons. Paraplegia 22: 311-315.

5 Brindley GS (1984) The fertility of men with spinal cord injuries. Paraplegia 22: 337-348.

6 Iwatsubo E, Tanaka N, Takahashi K, Akatsu T (1986) Non-inflatable penile prosthesis for the management of urinary incontinence and sexual disability of patients with spinal cord injury. Paraplegia 24: 307-310.

7 Yarkong GM (1990) Enhancement of sexual function and fertility in spinal cord-injured males. Am J Phys Med Rehabil 69: 81-87.

8 Moberg E (1990) Surgical rehabilitation of the upper limb in tetraplegia. Paraplegia 28: 330-334.

9 Buntine JA, Johnstone BR (1988) The contributions of plastic surgery to care of the spinal cord injured patient. Paraplegia 26: 87-93.

10 Johnstone BR, Jordan CJ, Buntine JA (1988) A review of surgical rehabilitation of the upper limb in quadriplegia. Paraplegia 26: 317-339.

11 Hill VB, Davies WE (1988) A swing to intermittent clean self-catheterisation as a preferred mode of management of the neuropathic bladder for the dextrous spinal cord patient. Paraplegia 26: 405-412.

12 Winfield HN, Clayman RV, Chaussy CG, Weyman PJ, Fuchs GJ, Lupu AN (1988) Monotherapy of staghorn renal calculi: a comparative study between percutaneous nephrolithotomy and extracorporeal shock wave lithotripsy. J Urol 189: 895-899.

13 Leroy AJ, Segura JW, Williams HJ, Patterson DE (1987) Percutaneous renal calculus removal in an extracorporeal shock wave lithotripsy practice. J Urol 138: 703-706.

14 McRae P, Murray KHA, Nurse DE, Stephenson TP, Mundy AR (1987) Clam enterocystoplasty in the neuropathic bladder. $\mathrm{Br} J$ Urol 60: 523-525.

15 Brindley GS, Polkey CE, Rushton DN (1982) Sacral anterior root stimulators for bladder control in paraplegia. Paraplegia 20: 365-381.

16 Brindley GS, Rushton DN (1990) Long term follow-up for patients with sacral anterior root stimulator implants Paraplegia 28: 469-475.

17 Minns RJ, Sutton RA (in press) Carbon fibre pad insertion as a method of achieving soft tissue augmentation in order to reduce the liability to pressure sore development in the spinal injury patient.

18 Peckham PH (1987) Functional electrical stimulation: current status and future prospects of application to the neuromuscular system in spinal cord injury. Paraplegia 25: 279-288.

19 Maynard FM (1986) Early clinical experience with clonidine in spinal spasticity. Paraplegia 24: 175-182.

20 Barbeau H, Richards CL, Bedard PJ (1982) Action of cyproheptadine in spastic paraparetic patients. J Neurol Neurosurg Psychiatry 45: 923-926.

21 Loubser BS, Narayan RK, Sandin KJ, Donovan WH, Russell KD (1991) Continuous infusion of intrathecal baclofen: long-term effects on spasticity in spinal cord injury. Paraplegia 29: 48-64.

22 Betz RR, Gelman AJ, DeFilipp GJ, Mesgarzadeh M, Clancy M, Steel HH (1987) Magnetic resonance imaging (MRI) in the evaluation of spinal cord injured children and adolescents. Paraplegia 25: 92-99.

23 Perovitch M (1987) The application of magnetic resonance in spinal cord disorders. Paraplegia 25: $373-380$.

24 Bondurant FJ, Cotler HB, Kulkarni MV, McArdle CB, Harris JH Jr (1990) Acute spinal cord injury. A study using physical examination and magnetic resonance imaging. Spine 15: 161-168.

25 Sett P, Crockard HA (1991) The value of magnetic resonance imaging (MRI) in the follow-up management of spinal injury. Paraplegia 29: 396-410.

26 Lindsey RW, Dick W (1991) The fixateur interne in the reduction and stabilization of thoracolumbar spine fractures in patients with neurologic deficit. Spine 16(3S): S140-145.

27 Transfeldt EE, White D, Bradford DS, Roche B (1990) Delayed decompression in patients with spinal cord and cauda equina injuries of the thoracolumbar spine. Spine 15: 953-957.

28 Aebi M, Etter Chr, Kehl TH, Thalgott J (1988) The internal skeletal fixation system: a new treatment of thoracolumbar fractures and other spinal disorders. Clin Orthop 227: 30-43.

29 Bracken MB, Shepard MJ, Collins WF et al (1990) A randomized, controlled trial of methylprednisolone or naloxone in the treatment of acute spinal cord injury. $N$ Engl J Med 322: 1405-1411.

30 Geisler FH, Dorsey FC, Coleman WP (1991) Recovery of motor function after spinal-cord injury: a randomized, placebo-controlled trail with GM-1 ganglioside. N Engl J Med 1991: 324: 1829-1838.

31 Hamilton BB, Granger CV, Sherwin FS, Zielezny M, Tashman JS (1987) A uniform national data system for medical rehabilitation. In: Fuhrer MJ, ed. Rehabilitation Outcomes: Analysis and Measurement. Paul $\mathrm{H}$ Brookes, Baltimore: 137-147.

32 Whittle MW, Cochrane GM, Chase AP et al (1991) A comparative trial of two walking systems for paralysed people. Paraplegia 29: 97-102.

33 Shanmugasundaram TK (1988) The care of SCI patients in developing nations - can we stem the rot? Paraplegia 26: 10-11.

34 Wang D, Wu X, Shi G, Wang Y (1990) China's first total care unit for the spinal cord injured. Paraplegia 28: $318-320$. 